\title{
Unitary black hole radiation: Schwarzschild-global monopole background
}

\author{
Arpit Das $^{\mathrm{a}}$, Narayan Banerjee ${ }^{\mathrm{b}}$ \\ Department of Physical Sciences, Indian Institute of Science Education and Research Kolkata, Mohanpur, West Bengal 741246, India
}

Received: 3 July 2019 / Accepted: 12 August 2019 / Published online: 21 August 2019

(C) The Author(s) 2019

\begin{abstract}
Black hole radiation from an infinitesimally thin massive collapsing shell, possessing a global monopole charge, which in turn leads to a Schwarzschild black hole with a global monopole charge has been shown to be processed by a unitary evolution. The exterior metric of the collapsing shell is described by the global monopole (GM) metric. The analysis is performed using the Wheeler-deWitt formalism which gave rise to a Schrödinger-like wave equation. Existence of unitarity is confirmed from two independent lines of approach. Firstly, by showing that the trace of the square of the density matrix, of the outgoing radiation, from a quantized massless scalar field, is unity. Secondly, by proving that the conservation of probability holds for the wave function of the system.
\end{abstract}

\section{Introduction}

Recently, in an attempt to shed some light on the resolution of the information loss paradox [1-6], it has been shown by Das and Banerjee [7] that radiation from a collapsing charged shell is processed with a unitary evolution. This was achieved in a Reissner-Nordström background using the WheelerdeWitt formalism [8,9] and unitarity checks were carried out using two independent lines of approach, density matrix and conservation of probability. We extend the result as given in [7] by performing the same kind of analysis for a not asymptotically flat spacetime. We adopt the formalism and method of analysis from [7] and apply it to a global monopole background metric [10]. It was shown in [11] that a Schwarzschild black hole with a global monopole charge Hawking radiation is Planckian in nature. So, naturally it is a relevant theoretical question to investigate unitarity issues in such backgrounds. This is the primary motivation of this work.

\footnotetext{
a e-mail: ad13ms118@iiserkol.ac.in

b e-mail: narayan@iiserkol.ac.in
}

The present work shows that the process of black hole radiation, in a not asymptotically flat spacetime, is unitary. Saini and Stojkovic [12] worked with a not asymptotically flat spacetime before, specifically with an asymptotically AdS spacetime. However, the results obtained therein are based on numerical estimates. For not asymptotically flat spacetimes, our analysis and therby the results obtained from them are more robust as they are done analytically.

We work with a metric that includes a global monopole charge $\eta$. The Schwarzschild case as considered in [13], is recovered trivially as a special case by setting $\eta=0$.

In Sect. 2 we describe the global monopole metric. Section 3 contains the description of the model. The scalar field is discussed in Sect. 4. The unitarity is ascertained in Sect. 5. The last section includes a discussion of the results.

\section{The global monopole}

The metric for a Schwarzschild black hole with a global monopole charge $\eta$ is given in natural units as $[10,11,14]$,

$$
\begin{aligned}
d s_{G M}^{2}= & -\left(1-\eta^{2}-\frac{2 M}{r}\right) d t^{2}+\left(1-\eta^{2}-\frac{2 M}{r}\right)^{-1} d r^{2} \\
& +r^{2} d \Omega_{2}^{2}
\end{aligned}
$$

where, $\eta^{2}<<1$ and $M$ is the mass of the black hole. Note that the above metric is not asymptotically flat and even with $M=0$ the spacetime is not flat, as it has some non-zero curvature [11],

$R_{0}^{0}=R_{1}^{1}=0=R_{01}$,

and, $\quad R_{2}^{2} \propto \frac{\eta^{2}}{r^{2}}$, 
where the above terms are the components of the Ricci tensor. The observational signature of a global monopole is in the existence of a "solid angle deficit".

The event horizon is at,

$R_{G M}=\frac{2 M}{1-\eta^{2}}$.

Let us also give below the stress-energy tensor corresponding to the Global monopole field [14],

$T_{0}^{0}=T_{1}^{1}=\frac{\eta^{2}}{8 \pi r^{2}}$,

where we see that the total energy is divergent and so solutions of such form as Eq. (1) are unrealistic and perhaps appear in some instances of cosmic phase transition [10].

The surface gravity $\kappa$ for the metric as given in Eq. (1) is obtained by noting that the metric is of the form [15],

$$
\begin{aligned}
& d s^{2}=-f d t^{2}+f^{-1} d r^{2}+r^{2} d \Omega_{2}^{2}, \\
& \text { implying, } \kappa=\frac{f^{\prime}(r)}{2} \\
& \text { implying, } \kappa_{G M}=\frac{\left(1-\eta^{2}\right)^{2}}{4 M} \\
& \quad\left(\text { as, } f(r)=1-\eta^{2}-\frac{2 M}{r}\right) .
\end{aligned}
$$

where $\kappa_{G M}$ is the surface gravity for the global monopole metric.

The semi-classical study of the metric as given in Eq. (1) was done in [11] and it was show that the outgoing Hawking radiation is thermal possessing a Planck spectrum,

$N=\frac{1}{e^{8 \pi M \omega /\left(1-\eta^{2}\right)^{2}}-1}$

where $N$ is the number density of outgoing quanta of particles. The Hawking temperature is recovered to be,

$T_{G M}=\frac{\left(1-\eta^{2}\right)^{2}}{8 \pi M}$,

which can also be obtained from Eq. (8) using the Hawking relation $T_{H}=\frac{\kappa}{2 \pi}$ (which holds here too).

\section{The model}

In our model we have an infinitesimally thin massive collapsing spherical shell with a global monopole charge $[11,14]$, whose background metric is $g_{\mu \nu}$. There is also a massless scalar field $\Phi$ whose dynamics we shall study. We assume that $\Phi$ couples to the gravitational field (which originates from the presence of a non-trivial background metric). However, $\Phi$ does not directly couple to the shell. An asymptotic observer, at the future null infinity, is present to detect the outgoing flux with a detector and by assumption does not interact with the "shell-metric-scalar" system. Hence, the observer does not significantly affect the evolution of the system and similarly for the system vis-a-vis the observer. The action for the whole system is then given by [16],

$$
\begin{aligned}
S_{\text {tot }}= & \int d^{4} x \sqrt{-g}\left[-\frac{\mathcal{R}}{16 \pi}+\frac{1}{2}\left(\partial_{\mu} \Phi\right)^{2}\right]-\sigma \int d^{3} \xi \sqrt{-\gamma} \\
& +S_{o b s}
\end{aligned}
$$

where the first term denotes the usual Einstein-Hilbert term for the background metric $g_{\mu \nu}$, the second term represents the action for the massless scalar field, the third term represents the shell's action in terms of its world-volume coordinates $\xi^{a}(a=0,1,2), \sigma$ is the tension of the shell (or, the shell's proper energy density per unit surface area) and $\gamma_{a b}$ is the shell's induced world-volume metric, given by,

$\gamma_{a b}=g_{\mu \nu} \partial_{a} X^{\mu} \partial_{b} X^{\nu}$,

where $X^{\mu}\left(\xi^{a}\right)$ determines the location of the shell. The Roman indices run over the internal world-volume coordinates $\xi^{a}(a=0,1,2)$ while the Greek indices run over the usual spacetime coordinates.

The last term $S_{o b s}$ represents the action for the observer.

\subsection{Spacetime foliation-GM coordinates}

The mass and the global monopole charge is confined in an infinitesimally thin shell [14], as per our considerations. So that for an exterior observer the distribution would be spherical. However, the inside of the shell would be empty and would be described by the Minkowski metric. The exterior of the shell is described by a global monopole metric. Thus, we have,

$$
\begin{aligned}
d s_{\text {out }}^{2}= & -\left(1-\eta^{2}-\frac{2 M}{r}\right) d t^{2} \\
& +\left(1-\eta^{2}-\frac{2 M}{r}\right)^{-1} d r^{2}+r^{2} d \Omega_{2}^{2},
\end{aligned}
$$

$d s_{i n}^{2}=-d T^{2}+d r^{2}+r^{2} d \Omega_{2}^{2}$,

$d s_{\text {on-shell }}^{2}=-d \tau^{2}+r^{2} d \Omega_{2}^{2}$,

for $r>R(t), r<R(t)$ and $r=R(t)$ respectively. Here $r$ is the radial coordinate. So $r=R(t)$ describes the collapsing shell and $R:=R(t)$ is the radius of the shell. $T, \tau$ and $t$ are the time coordinate inside the shell, proper time on the shell and 
time coordinate of the exterior observer respectively. $d \Omega_{2}^{2}$ is the standard $S^{2}$ metric.

An important consideration to observe here is that since the above GM coordinates would lead to a coordinate singularity, at $R=R_{G M}$ (the event horizon), we might face trouble using this for our analysis. However, observe that from the point of view of an asymptotic observer, the event horizon is an infinitely red shifted surface. So, the observer can only observe the collapse of the shell approaching its event horizon in infinite time as per his time $t$. Thus, the analysis would happen upto this limit which is relevant from an asymptotic viewpoint and the GM coordinates are well behaved upto this limit, that is just outside the event horizon.

Similar to [7], we consider timelike unit vectors $u^{\alpha}:=$ $\frac{d x_{\text {out }}^{\alpha}}{d \tau}$ and $v^{\alpha}:=\frac{d x_{\text {in }}^{\alpha}}{d \tau}$, for $d s_{\text {out }}^{2}$ and $d s_{\text {in }}^{2}$ respectively. From their normalization, that is, $u^{\alpha} u_{\alpha}=-1$ and $v^{\alpha} v_{\alpha}=-1$, one obtains, at $r=R(t), t_{\tau}=\frac{\sqrt{E+R_{\tau}^{2}}}{E}, T_{\tau}=\sqrt{1+R_{\tau}^{2}}$ and $T_{t}=\sqrt{E-(1-E) \frac{R_{t}^{2}}{E}}$. In the above expressions, a subscript indicates a differentiation w.r.t. that particular coordinate. $x_{\text {out }}^{\alpha}$ and $x_{\text {in }}^{\alpha}$ are the coordinates pertaining to $d s_{\text {out }}^{2}$ and $d s_{\text {in }}^{2}$ respectively. Also, $E:=1-\eta^{2}-\frac{2 M}{R(t)}$.

\subsection{Mass of the shell}

According to Israel's formulation [14,17,18], the mass $M$ of the shell can be obtained as,

$M=4 \pi \sigma R^{2}\left[\sqrt{1+R_{\tau}^{2}}-2 \pi \sigma R\right]-\frac{\eta^{2} R}{2}$,

We shall show below that $M$ would turn out to be a constant of motion. So, there would be no conflict with the fact that $M$ is a constant of integration in the metric and can be identified as the mass of the shell. Similar to the results given in [19], one can write,

$$
\frac{R_{\tau \tau}}{\alpha}=\frac{\eta^{2}}{8 \pi \sigma R^{2}}+6 \pi \sigma-\frac{2 \alpha}{R}, \quad\left(\text { where }, \alpha:=\sqrt{1+R_{\tau}^{2}}\right) .
$$

Now, using Eqs. (16) and (17),

$$
\begin{aligned}
M_{\tau}= & R_{\tau}\left[8 \pi \sigma R(\alpha-2 \pi \sigma R)-\frac{\eta^{2}}{2}\right] \\
& +R_{\tau}\left[4 \pi \sigma R^{2}\left(\frac{\eta^{2}}{8 \pi \sigma R^{2}}-\frac{2 \alpha}{R}+6 \pi \sigma-2 \pi \sigma\right)\right]=0 .
\end{aligned}
$$

Thus, we see that $M$ is a constant of motion.

Since, we have proven that $M$ is a constant of motion, we can have the following identification,

$\mathcal{H}_{\text {shell }} \equiv M$, where $\mathcal{H}_{\text {shell }}$ is the Hamiltonian of the shell. $\mathcal{H}_{\text {shell }}$ is to be treated classically for our analysis.

\subsection{Action for the shell}

The shell's action is given as,

$S_{\text {shell }}=-\int d T\left[4 \pi \sigma R^{2}\left[\sqrt{1-R_{T}^{2}}-2 \pi \sigma R\right]-\frac{\eta^{2} R}{2}\right]$.

The Lagrangian corresponding to the shell's action yields the conjugate momentum as,

$\Pi_{\text {shell }}=\frac{\partial \mathcal{L}_{\text {shell }}}{\partial R_{T}}=4 \pi \sigma R^{2}\left(\frac{R_{T}}{\sqrt{1-R_{T}^{2}}}\right)$.

Now the Hamiltonian is,

$$
\begin{aligned}
\mathcal{H}_{\text {shell }} & =\Pi_{\text {shell }} R_{T}-\mathcal{L}_{\text {shell }} \\
& =4 \pi \sigma R^{2}\left[\sqrt{1+R_{\tau}^{2}}-2 \pi \sigma R\right]-\frac{\eta^{2} R}{2} .
\end{aligned}
$$

$\mathcal{H}_{\text {shell }}$ as obtained above matches with $M$ as expressed in Eq. (16). Hence, the action in Eq. (19) is consistent (since, this action gives the correct $\mathcal{H}_{\text {shell }}$ as expressed in Eq. (18)). Now let us consider $S_{\text {shell }}$ in terms of time $t$, (using the expression for $T_{t}$ ),

$$
\begin{aligned}
S_{\text {shell }}= & -\int d t\left[4 \pi \sigma R^{2}\left[\sqrt{E-\frac{R_{t}^{2}}{E}}\right]\right] \\
& +\int d t\left[4 \pi \sigma R^{2}\left[2 \pi \sigma R \sqrt{E-\frac{1-E}{E} R_{t}^{2}}\right]\right] \\
& +\int d t\left[\frac{\eta^{2} R}{2} \sqrt{E-\frac{1-E}{E} R_{t}^{2}}\right]
\end{aligned}
$$

Let us also consider the conjugate momentum and Hamiltonian in terms of $t$,

$$
\begin{aligned}
\Pi_{\text {shell }}= & \frac{\partial \mathcal{L}_{\text {shell }}}{\partial R_{t}} \\
= & \frac{4 \pi \sigma R^{2} R_{t}}{\sqrt{E}}\left[\frac{1}{\sqrt{E^{2}-R_{t}^{2}}}-\frac{2 \pi \sigma R(1-E)}{\sqrt{E^{2}-(1-E) R_{t}^{2}}}\right] \\
& -\frac{4 \pi \sigma R^{2} R_{t}}{\sqrt{E}}\left[\frac{\eta^{2}(1-E)}{8 \pi \sigma R \sqrt{E^{2}-(1-E) R_{t}^{2}}}\right],
\end{aligned}
$$




$$
\begin{aligned}
\mathcal{H}_{\text {shell }}= & \Pi_{\text {shell }} R_{t}-\mathcal{L}_{\text {shell }} \\
= & 4 \pi \sigma E^{3 / 2} R^{2}\left[\frac{1}{\sqrt{E^{2}-R_{t}^{2}}}-\frac{2 \pi \sigma R}{\sqrt{E^{2}-(1-E) R_{t}^{2}}}\right] \\
& -4 \pi \sigma E^{3 / 2} R^{2}\left[\frac{\eta^{2}}{8 \pi \sigma R \sqrt{E^{2}-(1-E) R_{t}^{2}}}\right] .
\end{aligned}
$$

\subsection{Incipient limit}

We define the so-called incipient limit, $R \rightarrow R_{G M}$, as the limit when the radius of the shell approaches the event horizon. From Eqs. (23) and (24) we note that, as $R \rightarrow R_{G M}$,

$\Pi_{\text {shell }}=\frac{4 \pi \mu R^{2} R_{t}}{\sqrt{E} \sqrt{E^{2}-R_{t}^{2}}}$,

$\mathcal{H}_{\text {shell }}=\frac{4 \pi E^{3 / 2} \mu R^{2}}{\sqrt{E^{2}-R_{t}^{2}}}$,

where, $\mu:=\sigma\left(1-2 \pi \sigma R_{G M}-\frac{\eta^{2}}{8 \pi \sigma R_{G M}}\right)$. Then we have,

$\mathcal{H}_{\text {shell }}=\left[\left(E \Pi_{\text {shell }}\right)^{2}+E\left(4 \pi \mu R^{2}\right)^{2}\right]^{1 / 2} \equiv\left[q^{2}+m^{2}\right]^{1 / 2}$,

where $q^{2}:=\left(E \Pi_{\text {shell }}\right)^{2}$ and $m^{2}:=E\left(4 \pi \mu R^{2}\right)^{2}$.

$\mathcal{H}_{\text {shell }}$ as given in Eq. (27), is the Hamiltonian of a relativistic particle with a position dependent mass. This is how the shell behaves in the incipient limit as $R \rightarrow R_{G M}$. We shall show below that in this limit also, $\mathcal{H}_{\text {shell }}$ would turn out to be a constant of motion. Since, $\frac{d \mathcal{H}_{\text {shell }}}{d \tau}=\frac{\partial \mathcal{H}_{\text {shell }}}{\partial \tau}$, we have,

$\frac{d}{d \tau}\left(4 \pi \mu \frac{E^{3 / 2} R^{2}}{\sqrt{E^{2}-R_{t}^{2}}}\right)=0$

leading to, $\frac{E^{3 / 2} R^{2}}{\sqrt{E^{2}-R_{t}^{2}}}=\frac{\mathcal{H}_{\text {shell }}}{4 \pi \mu}=: h$ (a constant $)$,

(as $\tau$ doesn't appear explicitly in $\mathcal{H}_{\text {shell }}$ ).

These expressions can be arrived at independently using an alternative approach (see Appendix).

Classically, we have from Eq. (28) and from the expression of $T_{t}$,

$R_{t}= \pm E \sqrt{1-\frac{E R^{4}}{h^{2}}} \approx \pm E\left(1-\frac{1}{2} \frac{E R^{4}}{h^{2}}\right) \approx \pm E$ $\left(\right.$ as $\left.R \rightarrow R_{G M}\right)$,

$T_{t}=E \sqrt{1+(1-E) \frac{R^{4}}{h^{2}}}$,

where solving Eq. (29) in terms of $t$ will give us the classical behaviour of the shell as $R(t) \rightarrow R_{G M}$.

$E$ can be written as,

$E=\left(1-\eta^{2}\right)\left(1-\frac{R_{G M}}{R}\right)=\epsilon\left(1-\frac{R_{G M}}{R}\right)$,

where $\epsilon:=\left(1-\eta^{2}\right)$.

In the incipient limit, $E \rightarrow 0$ (as $R(t) \rightarrow R_{G M}$ ). Then, in this limit, $R_{t} \approx \pm E$. Now solving for $R(t)$ we get (from Eqs. (29) and (31)),

$\pm 1=\frac{1}{\epsilon} \frac{R}{R-R_{G M}} \frac{d R}{d t} \approx \frac{1}{\epsilon} \frac{R_{G M}}{R-R_{G M}} \frac{d R}{d t}$

(upto leading order)

integrating, $R_{G M} \ln \left(\frac{R_{f}-R_{G M}}{R_{0}-R_{G M}}\right)= \pm \epsilon t_{f}$

$$
\left(R_{0}:=R(0) \text { and } R_{f}:=R\left(t_{f}\right)\right)
$$

thus, $R_{f}=R_{G M}+\left(R_{0}-R_{G M}\right) e^{ \pm \epsilon t_{f} / R_{G M}}$,

where the lower limit of integration w.r.t. $t$ is $t=0$ and the upper limit is $t=t_{f}$.

Similar to as in [7], as $R_{f} \rightarrow R_{G M}$ and $t_{f}>0$ along with $\epsilon>0$ (as, $\eta^{2}<<1$ ), we observe that, $t_{f} \rightarrow \infty$. Thus, the negative sign for $R(t)$ describes a collapsing model in the incipient limit. Equation (32) also shows that from the viewpoint of an asymptotic observer, the formation of the event horizon takes infinite time implying that the event horizon is an infinite red shifted surface, which matches with the classical result, as stated earlier while choosing the GM coordinates.

\section{The scalar field $\Phi$}

The action for the scalar field $\Phi$ can be written as a sum of the actions,

$$
\begin{aligned}
S_{\Phi}= & \left.\left.S_{\Phi}\right)_{\text {in }}+S_{\Phi}\right)_{\text {out }} \\
= & 2 \pi \int d t\left[-\left(\partial_{t} \Phi\right)^{2}\left(\int_{0}^{R} d r r^{2} \frac{1}{T_{t}}\right)\right] \\
& +2 \pi \int d t\left[\left(\partial_{r} \Phi\right)^{2}\left(\int_{0}^{R} d r r^{2} T_{t}\right)\right]
\end{aligned}
$$




$$
\begin{aligned}
& +2 \pi \int d t\left[-\left(\partial_{t} \Phi\right)^{2}\left(\int_{R}^{\infty} d r r^{2} \frac{1}{1-\eta^{2}-\frac{2 M}{r}}\right)\right] \\
& +2 \pi \int d t\left[\left(\partial_{r} \Phi\right)^{2}\left(\int_{R}^{\infty} d r r^{2}\left(1-\eta^{2}-\frac{2 M}{r}\right)\right)\right]
\end{aligned}
$$

where the limits of the integration w.r.t. $r$ for $\left.S_{\Phi}\right)_{i n}$ are from 0 to $R$ and for $\left.S_{\Phi}\right)_{\text {out }}$ are from $R$ to $\infty$.

$T_{t} \rightarrow E$ (uptoleading order) in the incipient limit (from Eq. (30)). Thus,

$\lim _{R \rightarrow R_{G M}} \frac{T_{t}}{1-\eta^{2}-\frac{2 M}{r}}=\frac{R-\eta^{2} R-2 M}{r-\eta^{2} r-2 M} \frac{r}{R}=0$.

$T_{t}$ vanishes faster than $\left(1-\eta^{2}-\frac{2 M}{r}\right)$ in the limit $R \rightarrow$ $R_{G M}$. Thus, for the coefficients of $-\left(\partial_{t} \Phi\right)^{2}$, the $\frac{1}{T_{t}}$ term dominates. For the coefficients of $\left(\partial_{r} \Phi\right)^{2}$, the dominating term is $\left(1-\eta^{2}-\frac{2 M}{r}\right)$. Therefore, in the incipient limit,

$$
\begin{aligned}
S_{\Phi} \rightarrow & 2 \pi \int d t\left[-\frac{1}{E} \int_{0}^{R_{G M}} d r r^{2}\left(\partial_{t} \Phi\right)^{2}\right] \\
& +2 \pi \int d t\left[\int_{R_{G M}}^{\infty} d r r^{2}\left(1-\eta^{2}-\frac{2 M}{r}\right)\left(\partial_{r} \Phi\right)^{2}\right] .
\end{aligned}
$$

\subsection{Mode expansion for $\Phi$}

For $\Phi$, one can easily check from its equation of motion, that is $\partial^{2} \Phi=0$, that for $r<R(t)$ (from $\left.S_{\Phi}\right)_{i n}$ ),

$\frac{\partial^{2} \Phi}{\partial r^{2}}+\frac{2}{r} \frac{\partial \Phi}{\partial r}=\frac{1}{T_{t}^{2}} \frac{\partial^{2} \Phi}{\partial t^{2}}-\frac{T_{t t}}{T_{t}^{3}} \frac{\partial \Phi}{\partial t}$,

where $T_{t}$, along with its powers and derivatives w.r.t. $t$, are independent of $r$.

Similarly, for $r>R(t)$, we have (from $\left.S_{\Phi}\right)_{\text {out }}$ )),

$$
\begin{aligned}
& \left(1-\eta^{2}-\frac{2 M}{r}\right)^{2} \frac{\partial^{2} \Phi}{\partial r^{2}} \\
& +\frac{2(r-M)}{r^{2}}\left(1-\eta^{2}-\frac{2 M}{r}\right) \frac{\partial \Phi}{\partial r}=\frac{\partial^{2} \Phi}{\partial t^{2}}
\end{aligned}
$$

From Eqs. (35) and (36), we notice the following mode expansion (due to the separability property satisfied by the above equations),

$\Phi(r, t)=\sum_{k} a_{k}(t) f_{k}(r)$

where $a_{k}(t)$ are the modes and $f_{k}(r)$ are some real-valued smooth functions of $r$.
Now $S_{\Phi}$ in terms of modes $a_{k}$ is (as $R \rightarrow R_{G M}$ ),

$$
S_{\Phi}=\int d t \sum_{k, k^{\prime}}\left[-\frac{1}{2 E} \frac{d a_{k}}{d t} A_{k k^{\prime}} \frac{d a_{k^{\prime}}}{d t}+\frac{1}{2} a_{k} B_{k k^{\prime}} a_{k^{\prime}}\right]
$$

where $A_{k k^{\prime}}$ and $B_{k k^{\prime}}$ are defined as,

$$
\begin{aligned}
& A_{k k^{\prime}}:=4 \pi \int_{0}^{R_{G M}} d r r^{2} f_{k}(r) f_{k^{\prime}}(r), \\
& B_{k k^{\prime}}:=4 \pi \int_{R_{G M}}^{\infty} d r r^{2}\left(1-\eta^{2}-\frac{2 M}{r}\right) f_{k}^{\prime}(r) f_{k^{\prime}}^{\prime}(r),
\end{aligned}
$$

where, $f_{k}^{\prime}(r):=\frac{\partial f_{k}(r)}{\partial r}$. Observe that, both $A_{k k^{\prime}}$ and $B_{k k^{\prime}}$ are independent of $r$ and $t$ (as no $R(t)$ appears in them).

Following [7], we define the conjugate momenta, $\pi_{k} \mathrm{~s}$ (to the modes $a_{k}$ ) as,

$\pi_{k}:=\frac{\partial \mathcal{L}_{\Phi}}{\partial \dot{a}_{k}} \equiv-i \frac{\partial}{\partial a_{k}}$

where $\dot{a}_{k}:=\frac{d a_{k}}{d t}$, and from Eq. (38), we have (with $\mathcal{L}_{\Phi}$ defined as the Langrangian for $\Phi)$,

$\mathcal{L}_{\Phi}=\sum_{k, k^{\prime}}\left[-\frac{1}{2 E} \dot{a}_{k} A_{k k^{\prime}} \dot{a}_{k^{\prime}} d t+\frac{1}{2} a_{k} B_{k k^{\prime}} a_{k^{\prime}}\right]$,

$\mathcal{L}_{\Phi}=-\frac{1}{2 E}\left(\dot{\mathbf{a}}^{T} \mathbf{A} \dot{\mathbf{a}}\right)+\frac{1}{2}\left(\mathbf{a}^{T} \mathbf{B a}\right)$,

where $\mathbf{A}$ and $\mathbf{B}$ are non-singular linear operators, such that, $A_{k k^{\prime}} \in \mathbf{A}$ and $B_{k k^{\prime}} \in \mathbf{B}$ in the chosen bases, say $\left\{\dot{a}_{k}\right\}$ and $\left\{a_{k}\right\}$ respectively. In the basis $\left\{a_{k}\right\}, \mathbf{a}$ is a column vector, such that, $a_{k} \in \mathbf{a}$. One can similarly express $\dot{\mathbf{a}}$ in the basis $\left\{\dot{a}_{k}\right\}$.

For the Hamiltonian of $\Phi, \mathcal{H}_{\Phi}$, we obtain,

$$
\begin{aligned}
\mathcal{H}_{\Phi} & =\sum_{k} \pi_{k} \dot{a}_{k}-\mathcal{L}_{\Phi} \\
& =\sum_{k, k^{\prime}}\left[\frac{1}{2 E} \dot{a}_{k} A_{k k^{\prime}} \dot{a}_{k^{\prime}} d t+\frac{1}{2} a_{k} B_{k k^{\prime}} a_{k^{\prime}}\right] \\
= & \frac{E}{2}\left(\Pi^{T} \mathbf{A}^{-1} \Pi\right)+\frac{1}{2}\left(\mathbf{a}^{T} \mathbf{B a}\right),
\end{aligned}
$$

where $\Pi$ is a column vector, such that, $\pi_{k} \in \Pi$, in a chosen basis say $\left\{\pi_{k}\right\}$ and $\mathbf{A}^{-1}$ is the inverse of $\mathbf{A}$.

Following arguments similar to [7], note that, $\mathbf{B}$ and $\mathbf{A}$ are real and symmetric infinite dimensional matrices and hence are self-adjoint. Therefore, by the Spectral Theorem, there exists orthonormal bases of position space and momentum space consisting of respective eigenvectors of $\mathbf{B}$ and A. Furthermore, all the corresponding eigenvalues are real. Say, for instance, the bases for position space and momentum space 
are $\left\{b_{k}\right\}$ and $\left\{\dot{b}_{k}\right\}$ respectively (where, each $b_{k}$ is a linear combination of the original basis vectors $a_{k}$ and each $\dot{b}_{k}$ is a linear combination of the original basis vectors $\dot{a}_{k}$ ).

\subsection{The Schrödinger-like wave equation}

If we study the equation for one eigenvector $b \in\left\{b_{k}\right\}$, then our conclusion will be the same for all other eigenvectors (see [16]). So, we shall solve the Schrödinger-like wave equation for a wave functional $\Psi\left(\left\{b_{k}\right\}, t\right)$, which by the above assumption of equivalence is now a wave function $\psi(b, t)$. Therefore, $\psi(b, t) \equiv \Psi\left(\left\{b_{k}\right\}, t\right)$. Hence, using Eq. (43), we write the Schrödinger-like wave equation (for a single eigenvector b) as,

$$
\left[-\left(1-\eta^{2}-\frac{2 M}{R}\right) \frac{1}{2 \alpha} \frac{\partial^{2}}{\partial b^{2}}+\frac{1}{2} \beta b^{2}\right] \psi(b, t)=i \frac{\partial \psi(b, t)}{\partial t},
$$

where, $\alpha$ and $\beta$ are the eigenvalues of $\mathbf{A}$ and $\mathbf{B}$ respectively.

We define a new time parameter,

$\tilde{\eta}:=\int_{0}^{t} d t\left(1-\eta^{2}-\frac{2 M}{R}\right)$

leading to, $\frac{\partial \widetilde{\eta}}{\partial t}=E$,

and write Eq. (46) as

$\left[-\frac{1}{2 \alpha} \frac{\partial^{2}}{\partial b^{2}}+\frac{\beta}{2 E} b^{2}\right] \psi(b, \widetilde{\eta})=i \frac{\partial \psi(b, \widetilde{\eta})}{\partial \widetilde{\eta}}$.

Equation (49) becomes,

$\left[-\frac{1}{2 \alpha} \frac{\partial^{2}}{\partial b^{2}}+\frac{1}{2} \alpha \omega^{2}(\widetilde{\eta}) b^{2}\right] \psi(b, \widetilde{\eta})=i \frac{\partial \psi(b, \widetilde{\eta})}{\partial \widetilde{\eta}}$,

where, we have chosen to set $\widetilde{\eta}(t=0)=0$ and $\omega$ is defined as,

$\omega^{2}(\widetilde{\eta}):=\left(\frac{\beta}{\alpha}\right) \frac{1}{E}=: \frac{\omega_{0}^{2}}{E}$.

We observe that, Eq. (50) is a time dependent Simple Harmonic Oscillator (SHO) equation with $\omega(\widetilde{\eta})$ as the frequency.

In the incipient limit (using Eqs. (31) and (29)),

$\frac{d E}{d t}=\frac{2 M}{R^{2}} \frac{d R}{d t}=\epsilon \frac{2 M}{\epsilon R^{2}} \frac{d R}{d t} \approx-\epsilon E \frac{R_{G M}}{R_{G M}^{2}}=-\frac{\epsilon E}{R_{G M}}$.

Integrating Eq. (52) w.r.t. $t$ one gets (as $R \rightarrow R_{G M}$ ),

$E=1-\eta^{2}-\frac{2 M}{R(t)} \sim e^{-\epsilon t / R_{G M}}$.
From Eq. (53) we see that at late times, $1-\eta^{2}-\frac{2 M}{R(t)} \sim$ $e^{-\epsilon t / R_{G M}}$. Since we are interested in the incipient limit, that is, in late times of the collapsing process, we can choose the behaviour of $R(t)$ at early times as per our convenience for simplifying the calculations. Therefore, we choose both past and future behaviour of $R(t)$ to be stationary. Hence, we can take the metric to be flat for all $t \in(-\infty, 0)$. Stationarity in future can be achieved by taking a cut-off time $t_{f}$ for the collapse and then allowing $t_{f} \rightarrow \infty$, thus going into the continual collapse case till the black hole is formed. Therefore,

$E= \begin{cases}1, & \text { for } t \in(-\infty, 0) \\ e^{-\epsilon t / R_{G M}}, & \text { for } t \in\left(0, t_{f}\right) \\ e^{-\epsilon t_{f} / R_{G M}}, & \text { for } t \in\left(t_{f}, \infty\right)\end{cases}$

The above choice of $R(t)$ may seem quite problematic as $\frac{d R}{d t}$ is discontinuous at 0 and $t_{f}$, but references $[16,20]$ show that the particle production by the collapsing shell happens in the range, $0<t<t_{f}$ and in the $t_{f} \rightarrow \infty$ regime, all the solutions obtained are smooth and well-behaved. Therefore with the above considerations, the wavefunction $\psi$ would capture the whole collapse scenario, and in the limit of $t_{f} \rightarrow$ $\infty$ or $R(t) \rightarrow R_{G M}$, black hole formation sets in.

We note that, at early times, $t \in(-\infty, 0)$, the spacetime is Minkowski and therefore the initial vacuum states at $\mathcal{J}^{-}$ (past null infinity) are ${ }^{1}$ just the simple harmonic oscillator ground states (this can be seen from the form of Eq. (50), which with $\widetilde{\eta}=0$, is the SHO equation). Thus,

$\psi_{0}(b):=\psi(b, \tilde{\eta}=0)=\left(\frac{\alpha \omega_{0}}{\pi}\right)^{1 / 4} e^{-m \omega_{0} b^{2} / 2}$,

where $\psi_{0}(b)$ represents the SHO ground state and $\left\{\psi_{n}(b)\right\}$ will denote the SHO basis states at early times.

Equation (55) suggests that $\omega_{0}$ defined in Eq. (51) can be identified with the ground state frequency associated with the initial vacuum state.

With the help of Eq. (55), the exact solution to Eq. (50) is,

$\psi(b, \widetilde{\eta})=e^{i \chi(\widetilde{\eta})}\left[\frac{\alpha}{\pi \zeta^{2}}\right]^{1 / 4} \exp \left[i\left(\frac{\zeta \widetilde{\eta}}{\zeta}+\frac{i}{\zeta^{2}}\right) \frac{\alpha b^{2}}{2}\right]$

where $\zeta$ is the solution of the equation,

$\zeta \widetilde{\eta}+\omega^{2}(\widetilde{\eta}) \zeta=\frac{1}{\zeta^{3}}$

\footnotetext{
$\overline{1}$ One may see that the intuition behind identifying the states at $\mathcal{J}^{-}$ with the states at $t \in(-\infty, 0)$ actually comes from the fact that the observer is at $r \rightarrow \infty$. Now at an early time he is at $t \rightarrow-\infty$ which is $\mathcal{J}^{-}$and at late times he is at $t \rightarrow \infty$ which is $\mathcal{J}^{+}$.
} 
with the following initial conditions,

$\zeta(0)=\frac{1}{\sqrt{\omega_{0}}}$,

$\zeta_{\widetilde{\eta}}(0)=0$,

and, $\chi(\widetilde{\eta})$ is given by,

$\chi(\widetilde{\eta}):=-\frac{1}{2} \int_{0}^{\widetilde{\eta}} \frac{d \widetilde{\eta}}{\zeta^{2}\left(\widetilde{\eta}^{\prime}\right)}$.

Differential equations of the form Eq. (50) have been extensively studied in [21-25].

From Eqs. (51), (53) and (54), we have the following (for $t>0)$,

$\omega(\widetilde{\eta}(t))=e^{\epsilon t / 2 R_{G M}} \omega_{0}$.

Using Eqs. (48) and (61),

$\Omega(t)=\left(\left.\frac{\partial \widetilde{\eta}}{\partial t}\right|_{t>0}\right) \omega(\widetilde{\eta})=e^{-\epsilon t / 2 R_{G M}} \omega_{0}$

where $\Omega(t)$ is defined to be the frequency w.r.t. time $t$.

We note that at early times $\left(\mathcal{J}^{-}\right)$, the states are the initial vacuum states of SHO, as described by $\psi_{0}(b)$. With time, the frequency of the states $\Omega(t)$ evolve, as per Eq. (62), and more and more states get excited. Finally, when the observer measures them at $\mathcal{J}^{+}$(future null infinity), that is for some $t \in\left(t_{f}, \infty\right)$, we have the following mode expansion (following the evolution $n$ the Schrödinger picture [26]),

$\psi(b, t)=\sum_{n} c_{n}(t) \phi_{n}(b)$,

where $c_{n}(t)$ represent the probability amplitudes. The final SHO states $\left\{\phi_{n}(b)\right\}$ are with the frequency $\Omega_{f}=\Omega\left(t_{f}\right)$ (a constant), given by,

$\phi_{n}(b)=\left(\frac{\alpha \Omega_{f}}{\pi}\right)^{1 / 4} \frac{e^{-\alpha \Omega_{f} b^{2} / 2}}{\sqrt{2^{n} n !}} H_{n}\left(\sqrt{\alpha \Omega_{f}} b\right)$,

where $H_{n}$ are the Hermite polynomials. Observe that,

$\Omega\left(t_{f}\right)=e^{-\epsilon t_{f} / 2 R_{G M}} \omega_{0} ;$

$c_{n}$ can be computed from an overlap integral as (see Appendix), $c_{n}=\left\{\begin{array}{lr}\frac{(-1)^{n / 2} e^{i x}}{\left(\Omega_{f} \zeta^{2}\right)^{1 / 4}} \sqrt{\frac{2}{P}}\left(1-\frac{2}{P}\right)^{n / 2} \frac{(n-1) ! !}{\sqrt{n !}}, & \text { for even } n \\ 0, & \text { for } \text { odd } n,\end{array}\right.$

where $P:=1-\frac{i}{\Omega_{f}}\left(\frac{\zeta \tilde{\zeta}}{\zeta}+\frac{i}{\zeta^{2}}\right)$.

\section{Unitarity}

\subsection{Density matrix approach}

We shall now calculate the density matrices, $\hat{\rho}_{i}$ and $\hat{\rho}_{f}$, for the initial $\left(\mathcal{J}^{-}\right)$and the final $\left(\mathcal{J}^{+}\right)$states respectively. $\hat{\rho}_{i}$ and $\hat{\rho}_{f}$ can be written as (see $\left.[12,13]\right)$,

$\hat{\rho}_{i}=\sum_{m, n} l_{m} l_{n}^{*}\left|\psi_{m}\right\rangle\left\langle\psi_{n}\right|$,

$\hat{\rho}_{f}=\sum_{m, n} c_{m} c_{n}^{*}\left|\phi_{m}\right\rangle\left\langle\phi_{n}\right|$

where, $l_{n}$ and $c_{n}$ are the probability amplitudes appearing in the intial and final states respectively.

Since initially the system was in the SHO eigenstates $\left\{\psi_{n}\right\}$ and the wavefunction was normalized, we obtain,

$\operatorname{Tr}\left(\hat{\rho}_{i}\right)=1$

From Eq. (66), with $\lambda:=\left|1-\frac{2}{P}\right|$, we have,

$$
\begin{aligned}
\operatorname{Tr}\left(\hat{\rho}_{f}\right) & =\sum_{\text {even } n}\left|c_{n}\right|^{2} \\
& =\frac{2}{\sqrt{\Omega_{f} \zeta^{2}}|P|} \sum_{\text {even } n} \frac{(n-1) ! !}{n !} \lambda^{n} \\
& =\frac{2}{\sqrt{\Omega_{f} \zeta^{2}}|P|} \frac{1}{\sqrt{1-\lambda^{2}}} \\
& =\frac{2}{\sqrt{\Omega_{f} \zeta^{2}}|P|} \frac{1}{\sqrt{1-\left|1-\frac{2}{P}\right|^{2}}}
\end{aligned}
$$

$P$ has been computed explicitly and used in Eq. (70) to obtain (see Appendix),

$\operatorname{Tr}\left(\hat{\rho}_{f}\right)=1$.

Equation (71) shows that the necessary condition for the unitary evolution of states holds. For the sufficient condition, we compute $\operatorname{Tr}\left(\hat{\rho}_{f}^{2}\right)$. From Eq. (68), 


$$
\hat{\rho}_{f}=\sum_{m, n} c_{m} c_{n}^{*}\left|\phi_{m}\right\rangle\left\langle\phi_{n}\right|
$$

leading to, $\hat{\rho}_{f}^{2}=\left(\sum_{m, n} c_{m} c_{n}^{*}\left|\phi_{m}\right\rangle\left\langle\phi_{n}\right|\right)\left(\sum_{i, j} c_{i} c_{j}^{*}\left|\phi_{i}\right\rangle\left\langle\phi_{j}\right|\right)$

$=\sum_{m, n, i, j} c_{m} c_{i} c_{n}^{*} c_{j}^{*}\left|\phi_{m}\right\rangle\left\langle\phi_{n} \mid \phi_{i}\right\rangle\left\langle\phi_{j}\right|$

$=\sum_{m, n, j} c_{m} c_{j}^{*}\left|c_{n}\right|^{2}\left|\phi_{m}\right\rangle\left\langle\phi_{j}\right|$

$=\sum_{m, j} c_{m} c_{j}^{*}\left|\phi_{m}\right\rangle\left\langle\phi_{j}\right|\left(\sum_{n}\left|c_{n}\right|^{2}\right)$

$=\sum_{m, j} c_{m} c_{j}^{*}\left|\phi_{m}\right\rangle\left\langle\phi_{j}\right|$

$\left(a s,\left(\sum_{n}\left|c_{n}\right|^{2}\right)=1 b y e q^{n}(71)\right)$

$=\hat{\rho}_{f}$.

Therefore, by Eq. (72) we get,

$\operatorname{Tr}\left(\hat{\rho}_{f}^{2}\right)=\operatorname{Tr}\left(\hat{\rho}_{f}\right)=1$.

Analytically, we have shown that the idempotency of the final density matrix holds indicating a pure quantum state to pure quantum state transition.

\subsection{Conservation of probability approach}

The probability current 4 -vector $J^{\mu}$ can be defined as,

$J^{0}=|\psi|^{2}$,

$\mathbf{J}=\frac{1}{2 \alpha i}\left[\psi^{*} \nabla \psi-\psi \nabla \psi^{*}\right]$.

As $b$ is an eigenfunction of $\mathbf{B}$, it is independent of the spatial coordinates $x^{i}$. Thus, we conclude that $\mathbf{J}=\mathbf{0}$. This further suggests,

$\nabla_{\mu} J^{\mu}=\frac{\partial|\psi|^{2}}{\partial t_{o b s}}$.

Writing $t_{o b s}=t$ (for the observer's time coordinate), we have (from Eq. (48)),

$\nabla_{\mu} J^{\mu}=\frac{\partial|\psi|^{2}}{\partial t}=\frac{\partial|\psi|^{2}}{\partial \widetilde{\eta}} \frac{\partial \widetilde{\eta}}{\partial t}=E \frac{\partial|\psi|^{2}}{\partial \widetilde{\eta}}$

For, $R \rightarrow R_{G M}, \nabla_{\mu} J^{\mu}=0 \quad($ as,$E \rightarrow 0)$

Again analytically, we have shown from (Eq. (77)), that probability is conserved in the system, in the incipient limit of black hole formation.

\section{Conclusion}

We showed analytically and comprehensively that the black hole radiation, for a spacetime which is not asymptotically flat, is processed with a unitary evolution. This is confirmed from the density matrix consideration as well as from the conservation of probability consideration.

The Schrödinger-like wave equations that we used bear resemblance to a minisuperspace version of Wheeler-deWitt equations [8]. Interestingly, such equations have a present resurgence, in the context of issues concerned with unitarity [27-29].

Saini and Stojkovic [13] had showed that black hole radiation is processed with a unitary evolution, for a Schwarzchild black hole, from the density matrix consideration. However, they had achieved their conclusion through numerical estimates. We worked with a more general, metric, the global monopole metric, and results for the Schwarzchild case is recovered from this by putting $\eta=0$.

The computations on unitarity are all in the incipient limit, the limit of black hole formation. Hence, it does not really take care of the complete black hole evaporation process. However, if unitarity is preserved in this limit, it should be valid at every instant of time.

In saying this, we further emphasize that, what we have shown in this paper is that black hole radiation is unitary in a not asymptotically flat background spacetime. The present result of unitarity in spacetime that is not asymptotically flat, together with the results obtained in [7] that the unitarity is preserved for a Reissner-Nordstrom metric which is not globally hyperbolic, settles the issue of conservation of unitarity in spherically symmetric, static $(1+3)$ dimensional spacetimes of the form as given in Eq. (6). It also deserves mention that similar results for a Schwarzschild backround obtained in [13] numerically, can be arrived at as a special case from both of these more involved examples. So the results are quite consistent, and should have significant implications towards the resolution of the information loss paradox.

Acknowledgements AD would like to thank the Department of Science and Technology, Government of India for providing the INSPIRESHE scholarship which helped immensely in this research work.

Data Availability Statement This manuscript has no associated data or the data will not be deposited. [Authors' comment: There is no data taken from any sources. The work is based on analytical calculations and that is given in the main text, or in the appendix in the paper itself.]

Open Access This article is distributed under the terms of the Creative Commons Attribution 4.0 International License (http://creativecomm ons.org/licenses/by/4.0/), which permits unrestricted use, distribution, and reproduction in any medium, provided you give appropriate credit to the original author(s) and the source, provide a link to the Creative Commons license, and indicate if changes were made.

Funded by $\mathrm{SCOAP}^{3}$. 


\section{Appendix}

Alternate motivation for $S_{\text {shell }}$

In this section, we present a different action than $S_{\text {shell }}$. We shall call it $S_{\text {new }}$. We shall further show that in the incipient limit it will give rise to $\mathcal{H}_{\text {shell }}$ and $\Pi_{\text {shell }}$. Since we know that the shell behaves like a relativistic particle, we define the new action to be,

$$
\begin{aligned}
S_{\text {new }}= & -\int d \tau M=-\int d T \frac{M}{T_{\tau}}, \\
= & -4 \pi \sigma \int d T R^{2}\left[1-2 \pi \sigma R \sqrt{1-R_{T}^{2}}\right] \\
& +\int d T \frac{\eta^{2} R}{2} \sqrt{1-R_{T}^{2}}, \\
= & -4 \pi \sigma \int d t R^{2} \\
& \times\left[\sqrt{E-\frac{1-E}{E} R_{t}^{2}}-2 \pi \sigma R \sqrt{E-\frac{R_{t}^{2}}{E}}\right] \\
& +\int d t \frac{\eta^{2} R}{2} \sqrt{E-\frac{R_{t}^{2}}{E}} .
\end{aligned}
$$

Then,

$$
\begin{aligned}
\mathcal{L}_{\text {new }}= & -4 \pi \sigma R^{2}\left[\sqrt{E-\frac{1-E}{E} R_{t}^{2}}-2 \pi \sigma R \sqrt{E-\frac{R_{t}^{2}}{E}}\right] \\
& +\frac{\eta^{2} R}{2} \sqrt{E-\frac{R_{t}^{2}}{E}}, \\
\Pi_{\text {new }}= & \frac{\partial \mathcal{L}_{\text {new }}}{\partial R_{t}} \\
= & \frac{4 \pi \sigma R^{2} R_{t}}{\sqrt{E}}\left[\frac{1-E}{\sqrt{E^{2}-(1-E) R_{t}^{2}}}-\frac{2 \pi \sigma R}{\sqrt{E^{2}-R_{t}^{2}}}\right] \\
& -\frac{\eta^{2} R}{2} \frac{R_{t}}{\sqrt{E} \sqrt{E^{2}-R_{t}^{2}}}, \\
\mathcal{H}_{\text {new }}= & \Pi_{\text {new }} R_{t}-\mathcal{L}_{\text {new }} \\
= & 4 \pi \sigma E^{3 / 2} R^{2}\left[\frac{1}{\sqrt{E^{2}-(1-E) R_{t}^{2}}}-\frac{2 \pi \sigma R}{\sqrt{E^{2}-R_{t}^{2}}}\right] \\
& -\frac{\eta^{2} R}{2} \frac{E^{3 / 2}}{\sqrt{E^{2}-R_{t}^{2}}} .
\end{aligned}
$$

In the incipient limit we have,

$$
\begin{aligned}
\mathcal{H}_{\text {new }} & =\frac{4 \pi E^{3 / 2} \mu R^{2}}{\sqrt{E^{2}-R_{t}^{2}}}, \\
\Pi_{\text {new }} & =\frac{4 \pi \mu R^{2} R_{t}}{\sqrt{E} \sqrt{E^{2}-R_{t}^{2}}},
\end{aligned}
$$

where, $\mu:=\sigma\left(1-2 \pi \sigma R_{G M}-\frac{\eta^{2}}{8 \pi \sigma R_{G M}}\right)$. Observe that these are the exact same equations we had obtained before in this incipient limit.

Computation of $c_{n}$

Now let us compute the $c_{n}$ 's explicitly. We know that,

$\psi(b, t)=\sum_{n} c_{n}(t) \phi_{n}(b)$

From the overlap integral we have,

$$
\begin{aligned}
c_{n}= & \int d b \phi_{n}^{*} \psi=\left(\frac{\alpha^{2} \Omega_{f}}{\pi^{2} \zeta^{2}}\right)^{1 / 4} \frac{e^{i \chi(\widetilde{\eta})}}{\sqrt{2^{n} n !}} \\
& \times \int d b \exp \left[-\frac{\alpha \Omega_{f} b^{2}}{2}+i\left(\frac{\zeta_{\tilde{\eta}}}{\zeta}+\frac{i}{\zeta^{2}}\right) \frac{\alpha b^{2}}{2}\right] H_{n} \\
& \times\left(\sqrt{\alpha \Omega_{f}} b\right),
\end{aligned}
$$

$$
\begin{aligned}
= & \left(\frac{1}{\Omega_{f} \pi^{2} \zeta^{2}}\right)^{1 / 4} \frac{e^{i \chi(\widetilde{\eta})}}{\sqrt{2^{n} n !}} \\
& \times \int d x \exp \left[-\frac{x^{2}}{2}+\frac{x^{2}}{2} \frac{i}{\Omega_{f}}\left(\frac{\zeta \widetilde{\eta}}{\zeta}+\frac{i}{\zeta^{2}}\right)\right] H_{n}(x) \\
& \left(w i t h, x:=\sqrt{\alpha \Omega_{f}} b\right), \\
= & \left(\frac{1}{\Omega_{f} \pi^{2} \zeta^{2}}\right)^{1 / 4} \frac{e^{i \chi(\widetilde{\eta})}}{\sqrt{2^{n} n !}} \int d x e^{-P x^{2} / 2} H_{n}(x) \\
& \left(w i t h, P:=1-\frac{i}{\Omega_{f}}\left(\frac{\zeta \widetilde{\eta}}{\zeta}+\frac{i}{\zeta^{2}}\right)\right) \\
= & \left(\frac{1}{\Omega_{f} \pi^{2} \zeta^{2}}\right)^{1 / 4} \frac{e^{i \chi(\widetilde{\eta})}}{\sqrt{2^{n} n !}} I_{n} \\
& \left(\text { with, } I_{n}:=\int d x e^{-P x^{2} / 2} H_{n}(x)\right) .
\end{aligned}
$$

To compute $I_{n}$, let us consider the following generating function for the $H_{n}(x)$,

$J(z)=\int d x e^{-P x^{2} / 2} e^{-z^{2}+2 z x}=\sqrt{\frac{2 \pi}{P}} e^{-z^{2}(1-2 / P)}$,

since, $e^{-z^{2}+2 z x}=\sum_{n=0}^{\infty} \frac{z^{n}}{n !} H_{n}(x)$, 
$\int d x e^{-P x^{2} / 2} H_{n}(x)=\left.\frac{d^{n}}{d z^{n}} J(z)\right|_{z=0}$,

thus, $I_{n}=\sqrt{\frac{2 \pi}{P}}\left(1-\frac{2}{P}\right)^{n / 2} H_{n}(0)$,

as, $H_{n}(0)= \begin{cases}(-1)^{n / 2} \sqrt{2^{n} n !} \frac{(n-1) ! !}{\sqrt{n !}}, & \text { for even } n \\ 0, & \text { for odd } n .\end{cases}$

Thus we have,

$c_{n}=\left\{\begin{array}{lr}\frac{(-1)^{n / 2} e^{i \chi}}{\left(\Omega_{f} \zeta^{2}\right)^{1 / 4}} \sqrt{\frac{2}{P}}\left(1-\frac{2}{P}\right)^{n / 2} \frac{(n-1) ! !}{\sqrt{n !}}, & \text { for even } n \\ 0, & \text { for odd } n .\end{array}\right.$

Explicit computation of $\operatorname{Tr}\left(\hat{\rho}_{f}\right)$

We know that,

$\operatorname{Tr}\left(\hat{\rho}_{f}\right)=\frac{2}{\sqrt{\Omega_{f} \zeta^{2}}|P|} \frac{1}{\sqrt{1-\left|1-\frac{2}{P}\right|^{2}}}$.

To calculate $P$ explicitly, let us give the solution of,

$\zeta \widetilde{\eta} \tilde{\eta}+\omega^{2}(\widetilde{\eta}) \zeta=\frac{1}{\zeta^{3}}$

as,

$\zeta=\frac{1}{\sqrt{\omega_{0}}} \sqrt{\widetilde{\epsilon}^{2}+\varepsilon^{2}}$

$\zeta \widetilde{\eta}=\frac{1}{\omega_{0} \zeta}\left(\widetilde{\epsilon} \widetilde{\epsilon}_{\tilde{\eta}}+\varepsilon \varepsilon \widetilde{\eta}\right)$

where in terms of Bessel's functions, we have,

$\widetilde{\epsilon}=\frac{\pi u_{0}}{2}\left[Y_{0}\left(2 \omega_{0}\right) J_{1}\left(u_{0}\right)-J_{0}\left(2 \omega_{0}\right) Y_{1}\left(u_{0}\right)\right]$,

$\varepsilon=\frac{\pi u_{0}}{2}\left[Y_{1}\left(2 \omega_{0}\right) J_{1}\left(u_{0}\right)-J_{1}\left(2 \omega_{0}\right) Y_{1}\left(u_{0}\right)\right]$,

$\widetilde{\epsilon}_{\tilde{\eta}}=-\pi \omega_{0}^{2}\left[Y_{0}\left(2 \omega_{0}\right) J_{0}\left(u_{0}\right)-J_{0}\left(2 \omega_{0}\right) Y_{0}\left(u_{0}\right)\right]$,

$\varepsilon \widetilde{\eta}=-\pi \omega_{0}^{2}\left[Y_{1}\left(2 \omega_{0}\right) J_{0}\left(u_{0}\right)-J_{1}\left(2 \omega_{0}\right) Y_{0}\left(u_{0}\right)\right]$,

where $u_{0}:=2 \omega_{0} \sqrt{1-\widetilde{\eta}}$.

Now substituting the definition of $P$ (Eq. (86)) in Eq. (89), we have (using Mathematica),
$\operatorname{Tr}\left(\hat{\rho}_{f}\right)=\frac{\left|\zeta^{2} \Omega_{f}\right|}{\sqrt{\zeta^{2} \Omega_{f}} \sqrt{-\Im\left[\zeta^{2} \Omega_{f}\right] \Re[\zeta \zeta \tilde{\eta}]+(1+\Im[\zeta \zeta \tilde{\eta}]) \Re\left[\zeta^{2} \Omega_{f}\right]}}$.

Now as $\Omega_{f}, \zeta$ and $\zeta_{\tilde{\eta}}$ are real (as is evident from Eqs. (91-96)), we get from Eq. (97),

$\operatorname{Tr}\left(\hat{\rho}_{f}\right)=1$.

\section{References}

1. S.W. Hawking, Commun. Math. Phys. 43, 199 (1975)

2. S.W. Hawking, Phys. Rev. D 14, 2460 (1976)

3. D. Wallace, Why black hole information loss is paradoxical. Gen. Relativ. Quantum Cosmol (2018). arXiv:1710.03783v2 [gr-qc]

4. S.D. Mathur, Class. Quant. Grav. 26, 224001 (2009)

5. J. Polchinski, The black hole information problem. High Energy Phys. Theory (2016) arXiv:1609.04036 [hep-th]

6. D.N. Page, Phys. Rev. Lett. 71, 3743-3746 (1993)

7. A. Das, N. Banerjee, Eur. Phys. J. C 79, 475 (2019)

8. B.S. DeWitt, Phys. Rev. 160, 1113 (1967)

9. J.A. Wheeler, Superspace and the nature of quantum geometrodynamics, in Batelle Recontres (Benjamin, New York, 1968)

10. M. Barriola, A. Vilenkin, Phys. Rev. Lett. 63, 341 (1989)

11. N. Dadhich, K. Narayan, U.A. Yajnik, Pramana J. Phys. 50, 307 (1998)

12. A. Saini, D. Stojkovic, Phys. Rev. D 97, 025020 (2018)

13. A. Saini, D. Stojkovic, Phys. Rev. Lett. 114, 111301 (2015)

14. K.G. Zloshchastiev, Phys. Rev. D 57, 4812 (1998)

15. E. Poisson, A Relativist's Toolkit: the Mathematics of Black-Hole Mechanics (Cambridge University Press, Cambridge, 2007)

16. T. Vachaspati, D. Stojkovic, L.M. Krauss, Phys. Rev. D 76, 024005 (2007)

17. W. Israel, Nuovo Cimento 44B, 1 (1966)

18. W. Israel, Nuovo Cimento A 51, 744 (1967)

19. C.A. López, Phys. Rev. D 38, 3662 (1988)

20. E. Greenwood, JCAP 1001, 002 (2010)

21. C.M.A. Dantas, I.A. Pedrosa, B. Baseia, Phys. Rev. A 45, 1320 (1992)

22. H.R. Lewis, J. Math. Phys. 9, 1976 (1968)

23. H.R. Lewis, W.B. Riesenfeld, J. Math. Phys 10, 1458 (1969)

24. I.A. Pedrosa, J. Math. Phys. 28, 2662 (1987)

25. M. Kolopanis, T. Vachaspati, Phys. Rev. D 87, 085041 (2013)

26. J.J. Sakurai, J. Napolitano, Modern Quantum Mechanics, 2nd edn. (Addison-Wesley, Redwood City, 2011)

27. S. Pal, N. Banerjee, Phys. Rev. D 90, 104001 (2014)

28. S. Pal, N. Banerjee, Phys. Rev. D 91, 044042 (2015)

29. S. Pal, N. Banerjee, J. Math. Phys. 57, 122502 (2016) 